\title{
Properties of road unevenness inducing the kinematical excitation of vehicles
}

\author{
Mária Kúdelčíková ${ }^{1, *}$ and Jozef Melcer ${ }^{1}$ \\ ${ }^{1}$ University of Žilina, Faculty of Civil Engineering, Univerzitná 8215/1, 01026 Žilina, Slovakia
}

\begin{abstract}
The submitted paper is devoted to the mapping of the surface road profile and to the mathematical description of unevenness in one vehicle track. Its statistical parameters are analyzed and the classification of the road into a category based on power spectral density of unevenness is made.
\end{abstract}

\section{Introduction}

Modeling of dynamic effect of moving load on the road is a serious engineering problem. Analysis of the problem implies: creation of computational models of vehicles, creation of computational models of roads, modeling of runway surface unevenness, method of solving equation of motion, processing and presentation of results. Surface unevenness represents an important role in this process, because it is the source of kinematic excitation of the vehicle and therefore the source of dynamic components of the contact forces $[1,2]$. The submitted paper is devoted to the mapping of the surface road profile, mathematical description of unevenness in one vehicle track, analyzing its statistical parameters and the classification of the road into a category based on power spectral density of unevenness.

\section{Unevenness mapping}

Unevenness mapping of the roadway was carried out by the prototype of new developed devices like vibrograph. The result of mapping was road profile in one vehicle track, Fig. 1.

Road profile in one vehicle track

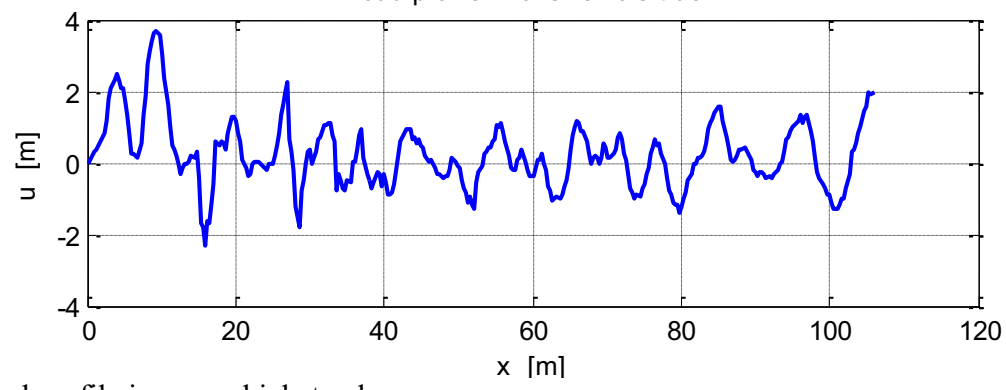

Fig. 1. Road profile in one vehicle track.

* Corresponding author: maria.kudelcikova@,fstav.uniza.sk 
It was mapped the section $105.9 \mathrm{~m}$ long in increments of $0.3 \mathrm{~m}$.

\section{Amplitude characteristics of unevenness}

The original record had 354 samples. The record was re-sampled. New record has $N=2^{13}$ $=8192$ samples. All other analyzes were performed with such re-sampled record. The following characteristics were calculated:

Mean value

$$
\bar{u}=\frac{1}{N} \sum_{i=1}^{N} u_{i}=0.232256 \mathrm{~mm} .
$$

Arithmetic mean deviation $R_{a}$

$$
R_{a}=\frac{1}{N} \sum_{i=1}^{N}\left|u_{i}-\bar{u}\right|=0.690666 \mathrm{~mm} .
$$

Root mean square average deviation $R_{q}$

$$
R_{q}=\sqrt{\frac{1}{N} \sum_{i=1}^{N}\left(u_{i}-\bar{u}\right)^{2}}=0.931226 \mathrm{~mm} .
$$

Dispersion $\sigma^{2}$

$$
\sigma^{2}=\frac{1}{N} \sum_{i=1}^{N}\left(u_{i}-\bar{u}\right)^{2}=0.867182 \mathrm{~mm}^{2} .
$$

Effective values - Root mean square value $R M S$

$$
\bar{u}=\sqrt{\frac{1}{N} \sum_{i=1}^{N} u_{i}^{2}}=0.959753 \mathrm{~mm} .
$$

Asymmetry coefficient $R_{s k}$

$$
R_{s k}=\frac{\sum_{i=1}^{N}\left(u_{i}-\bar{u}\right)^{3}}{N \cdot \sigma^{3}}=0.839111 .
$$

Kurtosis $R_{k u}$

$$
R_{k u}=\frac{\sum_{i=1}^{N}\left(u_{i}-\bar{u}\right)^{4}}{N \cdot \sigma^{4}}=4.788243 .
$$

Greatest depth of unevenness

$$
R_{v}=\min \left(u_{i}\right)=-2.327134 \mathrm{~mm} .
$$


The largest height of unevenness

$$
R_{p}=\max \left(u_{i}\right)=3.709063 \mathrm{~mm} .
$$

Overall height of the profile

$$
R_{t}=\left|R_{v}\right|+R_{p}=2.327134+3.709063=6.036197 \mathrm{~mm} .
$$

The histogram of amplitude distribution is shown in Fig. 2.

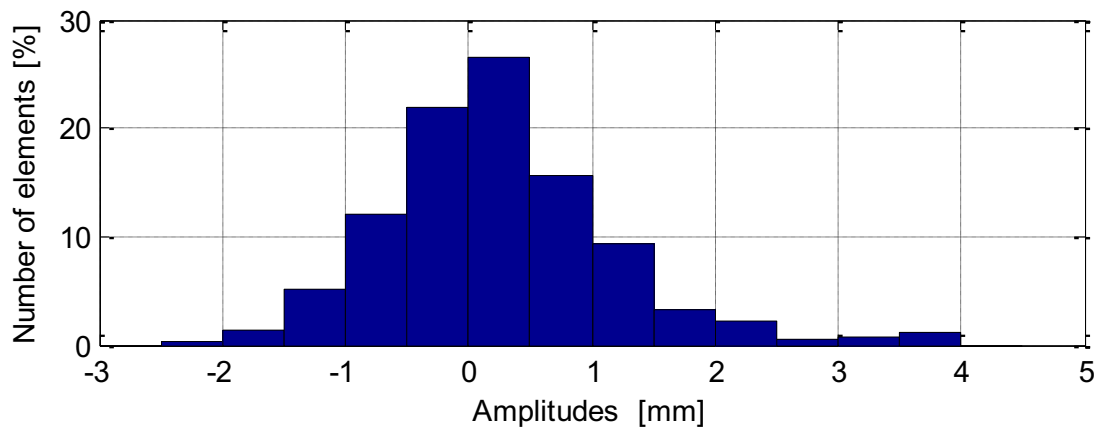

Fig. 2. Histogram of amplitude distribution.

\section{Spectral characteristics of unevenness}

Passing from length to frequency domain can be carried out by Fast Fourier Transform. To each real number $u_{k}$ it is assigned a complex number $U_{k}$ by the relation

$$
U_{k}=\sum_{n=1}^{N} u_{n} \cdot \mathrm{e}^{-2 \pi \cdot \mathrm{i} \cdot(k-1)(n-1) / N}, \text { for } 1 \leq k \leq N .
$$

$N$ is the number of samples of the record. The amplitude and phase angle are then calculated for each complex number, so the amplitude and phase spectra can be calculated for the record. There are a few possibilities how to present the amplitude spectrum:

Peak Amplitude $P A$

$$
\begin{gathered}
P A_{I}=\sqrt{\operatorname{Re}\left[U_{I}\right] \cdot \operatorname{Re}\left[U_{I}\right]+\operatorname{Im}\left[U_{I}\right] \cdot \operatorname{Im}\left[U_{I}\right]} / N, \text { for } I=0, \\
P A_{I}=2 \cdot \sqrt{\operatorname{Re}\left[U_{I}\right] \cdot \operatorname{Re}\left[U_{I}\right]+\operatorname{Im}\left[U_{I}\right] \cdot \operatorname{Im}\left[U_{I}\right]} / N, \quad I=1 \div(N / 2)-1
\end{gathered}
$$

Auto Spectrum $A S$

$$
A S_{I}=P A_{I} \cdot P A_{I}, \quad \text { for } I=0 \div(N / 2)-1 \text {. }
$$

Root Mean Square Amplitude RMSA

$$
\begin{gathered}
R M S A_{I}=P A_{I}, \quad \text { for } I=0, \\
R M S A_{I}=P A_{I} / \sqrt{2}, \quad \text { for } I=1 \div(N / 2)-1
\end{gathered}
$$


Power Spectrum PS

$$
\begin{gathered}
P S_{I}=A S_{I}, \quad \text { for } I=0, \\
P S_{I}=A S_{I} / 2, \quad \text { for } I=1 \div(N / 2)-1 .
\end{gathered}
$$

The spectrum is usually shown as a function of wavenumber $\Omega=2 \pi / L$ in $[\mathrm{rad} / \mathrm{m}]$ or as a function of wavelength $L$ in [m]. The PSD of unevenness $u$ as a function of $\Omega$ is shown in Fig. 3 and peak amplitude of unevenness $u$ as a function of $L$ is shown in Fig. 4.

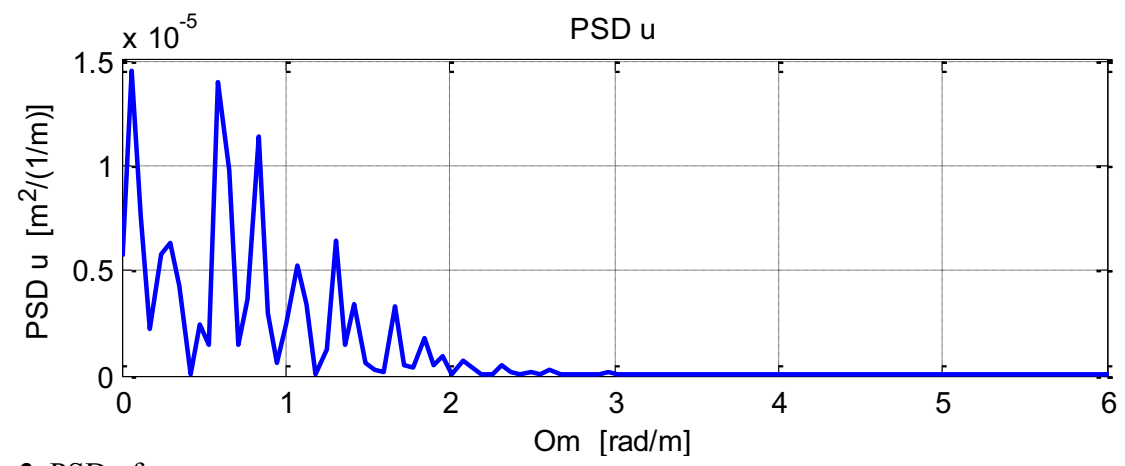

Fig. 3. PSD of unevenness $u$.

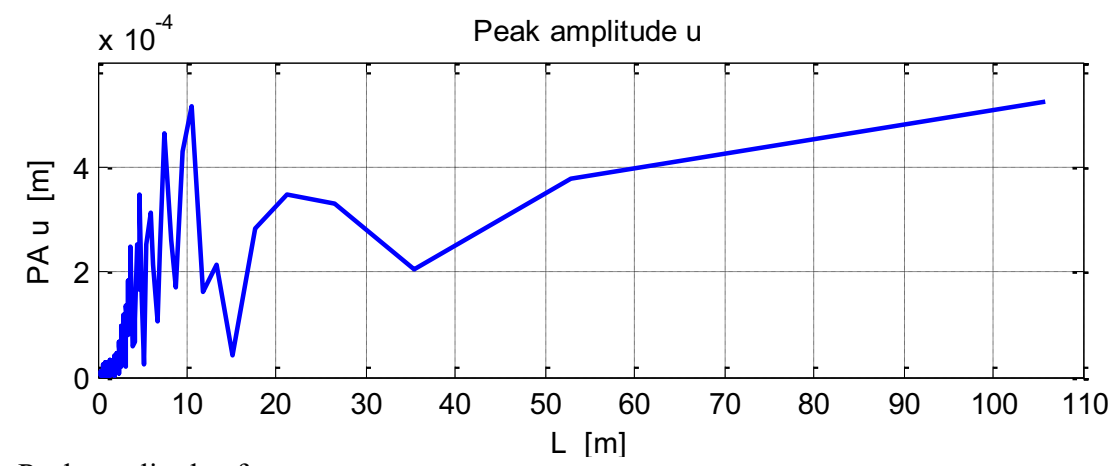

Fig. 4. Peak amplitude of unevenness $u$.

The standard ISO 8608 [3] classified the road under the PSD into 8 categories A $-\mathrm{H}$. Our road profile can be classified in the category B, Fig. 5.

\section{Conclusion}

Road unevenness represents the source of kinematic excitation of vehicle. It directly affects the vibration of the vehicle and the value of the dynamic components of the contact forces. The knowledge of real road profile and its amplitude and spectral characteristics is needed for classification of the road and for the numerical simulation of vehicle motion along the road. Our profile is $105.9 \mathrm{~m}$ long. Overall height of the profile is $6.03 \mathrm{~mm}$. The greatest depth and the largest height are -2.32 and $3.71 \mathrm{~mm}$, respectively. The profile contains the waves of wavelength $L$ in the interval $0.97-105.9 \mathrm{~m}$. By the Standard ISO 8608 the profile can be classified as category B. It can be used as input value for numerical simulation of motion of plane vehicle computing model along the road. 


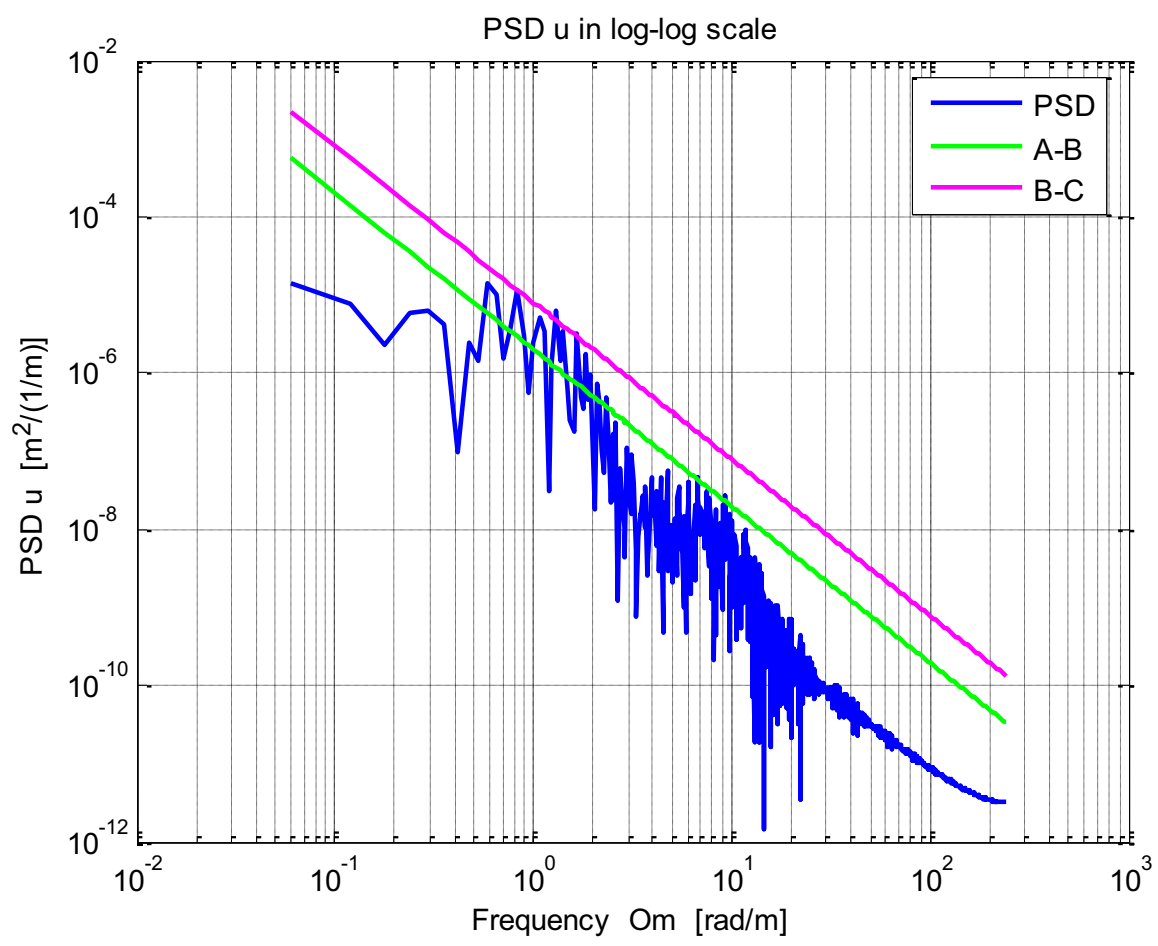

Fig. 5. Classification of unevenness $u$.

This work was supported by the Grant National Agency VEGA of the Slovak Republic, project number 1/0005/16.

\section{References}

1. G. Rill, Road vehicle dynamics, fundamentals and modeling (CRC Press, Taylor \& Francis Group, London, New York, 2012)

2. M. Decký, M. Kováč, Longitudinal unevenness of roads (EDIS, Žilina, 2014, in Slovak)

3. ISO 8608, Mechanical vibration - road surface profiles - reporting of measures data, International standard, (1995) 